\title{
Transcatheter Treatment of Complex Pulmonic and Aortic Valvular Disease Following Failed Ross Procedure
}

\author{
Mohammad Aziz ${ }^{1}$, Rajbir Sidhu², and Curtiss Stinis ${ }^{3}$ \\ ${ }^{1}$ Scripps Green Hospital \\ ${ }^{2}$ Scripps Memorial Hospital La Jolla \\ ${ }^{3}$ Scripps Clinic and Research Foundation
}

June 1, 2021

\begin{abstract}
A 63 year old male with congenital bicuspid aortic valve status post Ross procedure complicated by multiple sternotomies to treat a failed pulmonic homograft and pseudoaneurysm repair of both ventricular outflow tracts, bioprosthetic aortic valve replacement with subsequent endocarditis who presented with recurrent pulmonic/aortic vavlulopathy successfully treated with transcatheter intervention.
\end{abstract}

Transcatheter Treatment of Complex Pulmonic and Aortic Valvular Disease Following Failed Ross Procedure

Mohammad Aziz MD ${ }^{1}$, Rajbir Sidhu $\mathrm{MD}^{2}$, Curtiss T. Stinis MD ${ }^{3}$

${ }^{1}$ Department of Internal Medicine, Scripps Clinic, La Jolla, CA, USA

${ }^{2,3}$ Department of Cardiovascular Diseases, Scripps Clinic, La Jolla, CA, USA

Location: Performed at Scripps Memorial Hospital in La Jolla, CA, USAIndex: Bicuspid aortic valve, Ross Procedure, Pulmonary valve stenosisShort Title: Considerations in Percutaneous InterventionWord Count: 1647Corresponding Author: Mohammad Aziz Scripps Clinic, Division of Internal Medicine 10710 N. Torrey Pines Road La Jolla, CA, 92037, USA Tel: 818-614-8382 Email: Mohammad.azizmd@gmail.com

\begin{abstract}
A 63 year old male with congenital bicuspid aortic valve disease and a complex surgical history which includes a Ross procedure complicated by cardiac arrest requiring emergency coronary artery bypass graft surgery, multiple subsequent sternotomies to treat a failed pulmonic homograft and pseudoaneurysm repair of the left and right ventricular outflow tracts (LVOT/RVOT), bioprosthetic aortic valve replacement, and aortic valve endocarditis who presented with worsening heart failure symptoms secondary to bioprosthetic aortic valve failure and recurrent pulmonic valve stenosis successfully treated with transcatheter intervention.
\end{abstract}

\section{Key Clinical Message:}

Hemodynamic and technical considerations of transcatheter pulmonic and aortic valve replacement and future implications for possible repeat intervention.

\section{Funding statement:}

This project did not receive any funding.

\section{Author Contributions:}


MA drafted the manuscript.

RS made edits to manuscript and helped in drafting manuscript.

CS made edits to manuscript, helped in drafting manuscript and performed the procedure.

\section{Introduction}

The Ross Procedure is a surgical treatment which consists of using the patient's native pulmonic valve to replace the diseased aortic valve with heterograft pulmonic valve replacement. The procedure is typically performed in younger patients but is often associated with long term complications requiring recurrent intervention.

\section{Case Report}

\section{History of Presentation:}

A 63-year-old male presents with one year of progressive heart failure symptoms (NYHA Class III/IV). Clinical examination confirmed a IV/VI late peaking crescendo decrescendo murmur at the RUSB.

\section{Past Medical History:}

The patient underwent a Ross procedure in 2002 for congenital bicuspid aortic stenosis which was complicated by post-procedure cardiac arrest due to left main coronary obstruction. This was treated with emergent coronary bypass surgery utilizing left internal mammary artery (LIMA) to left anterior descending (LAD) and saphenous vein graft (SVG) to the left circumflex (LCx) coronary artery. The patient was subsequently found to have aortic valve paravalvular leak (PVL) with fistulization of the non-coronary cusp to the left ventricle and right coronary cusp to right ventricle requiring redo sternotomy for correction. In 2013, he developed severe pulmonary homograft stenosis as well as a pseudoaneurysm of the LVOT necessitating repeat sternotomy with pulmonary homograft replacement and concomitant bioprosthetic aortic valve replacement. In 2014, the patient underwent another sternotomy for patch repair of recurrent LVOT/RVOT pseudoaneurysm which was complicated by aortic valve insufficiency requiring repeat aortic valve replacement with a $21 \mathrm{~mm}$ Edwards Magna Ease valve. His post-op course was complicated by recurrent episodes of endocarditis and development of an annular abscess resulting in multiple hospital admissions in 2014, 2015 and 2017 for intravenous antibiotic treatment.

\section{Investigations:}

Transthoracic echocardiography (TTE) was significant for severe bioprosthetic aortic valve stenosis with moderate to severe paravalvular regurgitation (peak velocity $3.7 \mathrm{~m} / \mathrm{s}$, DVI 0.24 , acceleration time $>100 \mathrm{~ms}$ with mean gradient $35 \mathrm{mmHg}$ ) (Figure A) . Left ventricular ejection fraction was $46 \%$. Additionally, pulmonic valve stenosis was identified with peak/mean gradient of $66 / 41 \mathrm{~mm} \mathrm{Hg}$ and max velocity $4.0 \mathrm{~m} / \mathrm{s}$. Angiography demonstrated occluded LIMA to LAD graft with the native LAD supplied by the native left main, and total occlusion of the native LCX with patent SVG to the LCx. A right heart catheterization (RHC) was remarkable for severely elevated right ventricular systolic pressure of $102 \mathrm{~mm} \mathrm{Hg}$ (Figure B).Pulmonary artery pressure (PAP) was $32 / 15 \mathrm{~mm} \mathrm{Hg}$ with mean PAP of $22 \mathrm{~mm} \mathrm{Hg}$.

\section{Management:}

Endovascular pulmonic valve replacement was undertaken by first performing serial balloon dilatation of the stenotic homograft using $16 \mathrm{~mm}, 18 \mathrm{~mm}$, and $20 \mathrm{~mm}$ balloons. Because of the increased risk of coronary compression with transcatheter pulmonic valve interventions, patency of the SVG to LCx and left main coronary arteries was assessed during each balloon inflation using selective angiography. During inflation of the $20 \mathrm{~mm}$ balloon there was partial angiographic compromise of the left main coronary artery noted, indicating that this would be the maximal safe diameter obtainable for transcatheter pulmonic valve implantation(Figure C). Subsequent angiography demonstrated a small area of contained perforation of the homograft. As such, a neoconduit within the homograft was created by implantation of an $18 \mathrm{~mm} \times 28$ $\mathrm{mm}$ Cheatham Platinum (CP) covered stent followed by deployment of a $10 \mathrm{~mm}$ x $29 \mathrm{~mm}$ Palmaz stent; 
post-dilated to $20 \mathrm{~mm}$ for additional reinforcement and resistance to elastic recoil (Figure D). A $20 \mathrm{~mm}$ Edwards Sapien S3 valve was then deployed inside the neoconduit. Final pulmonary artery angiography showed no significant pulmonic regurgitation(Figure E). Right ventricular pressure improved to $41 / 4 \mathrm{~mm}$ $\mathrm{Hg}$

\section{(Figure F).}

Two weeks later, the patient underwent valve-in-valve (VIV) transcatheter aortic valve replacement (TAVR) utilizing a $23 \mathrm{~mm}$ Edwards Sapien Ultra valve under transesophgeal echocardiographic (TEE) guidance. The valve was deployed somewhat more ventricular than is typical in order to utilize the valve's sealing skirt to seal the large area of PVL involving the superior LVOT. Following valve deployment, additional balloon postdilatation was performed using a 22mm Tru Dilatation Balloon (Bard) to deliberately fracture and enlarge the bioprosthetic surgical valve to further seal the large area of PVL and to assure optimal transvalvular hemodynamics (Figure G). The pressure gradient across the aortic valve resolved after TAVR implantation (Figure H). Final TEE imaging revealed near total resolution of both PVL and aortic stenosis (Figure I).

\section{Discussion}

Although the patient met class I indications for aortic and pulmonic valve replacement, we instead proceeded with an endovascular approach to address both valvular pathologies given his high risk for repeat traditional surgery given his multiple previous sternotomies and surgical complications. We elected to proceed with intervention for pulmonic stenosis first given that right ventricular dysfunction is typically more recalcitrant to reversal than is left ventricular dysfunction due to limited medical therapies; and due to concern for potential worsening of left sided output due to inadequate preload should right ventricular dysfunction develop.

Ideal transcatheter pulmonic intervention involves implanting a valve large enough to eliminate the hemodynamic stress placed on the right ventricle while simultaneously avoiding catastrophic rupture of the stenotic pulmonic conduit and/or coronary artery compression. In this case, we were unable to obtain surgical records confirming the original pulmonic conduit size. Therefore, we proceeded with serial balloon dilation to determine the maximal feasible neoconduit size. Because of the risk of coronary compression, it is critical to perform coronary angiography during each balloon inflation to assure that coronary compression is not occurring. The maximal allowable size of the neoconduit is therefore typically limited by the largest diameter balloon used that does not lead to coronary compression.

In this case, balloon dilatation lead to a small contained perforation of the surgical pulmonic conduit, and thus a CP covered stent was deployed to seal the contained perforation. Although the CP stent sealed the perforation, it does not have adequate structural radial support to resist elastic recoil. Therefore, the addition of the Palmaz stent was necessary to add structural integrity to the neoconduit. With the proper scaffolding in place, the Edwards valve could then be deployed.

The Aortic valve intervention presented the challenge of treating bioprosthetic valve stenosis with concomitant moderate to severe PVL. After valve deployment, post-dilation bioprosthetic valve fracture (BVF) and enlargement of the surgical valve lead to sealing of the PVL and improvement in overall hemodynamics. Given the patient's young age, we prioritized treatment of PVL at the LVOT level by placing a Sapien Ultra valve slightly more ventricular than would be typical, followed by BVF. This strategy avoided placement of a vascular plug which can increase the risk of complications with future TAVR in TAVR procedures by deforming during valve expansion leading to ostial right coronary artery (RCA) obstruction or migration.

Follow-up:

At one month follow up, the patient reported symptomatic improvement. At 3 months, he was exercising more than 20 minutes per day at the gym and reported walking up several flights of stairs without difficulty. His heart failure symptoms resolved, and diuretic therapy was discontinued.

\section{Conclusion}


This case highlights the utility of transcatheter techniques in addressing multivalvular pathology including pulmonary valve in valve intervention in patients with symptomatic pulmonary valve stenosis and TAVR for severe aortic stenosis who are not ideal surgical candidates. Techniques for safe and optimal valve implantation and achieving optimal hemodynamics are outlined along with implications for future intervention.

\section{Author Disclosures}

Dr. Stinis has served as a consultant and proctor for Edwards Lifesciences

\section{Abbreviations and Acronyms}

LVOT Left ventricular outflow tract

RVOT Right ventricular outflow tract

LIMA Left internal mammary artery

LAD Left anterior descending

SVG Saphenous vein graft

LCx Left Circumflex

PVL Paravalvular leak

TTE Transthoracic echocardiography

RHC Right heart catheterization

PAP Pulmonary artery pressure

CP Cheatham Platinum

VIV Valve in valve

TAVR Transcatheter aortic valve replacement

TEE Transesophageal echocardiogram

BVF Bioprosthetic valve fracture

RCA Right coronary artery

\section{Figure Legends}

FIGURE A: Initial transthoracic echocardiographic parasternal short axis image demonstrating color doppler of large jet of paravalvular aortic regurgitation.

FIGURE B: Right heart catheterization (RHC) with severely elevated right ventricular systolic pressure of $102 \mathrm{~mm} \mathrm{Hg}$.

FIGURE C: Angiographic image performed during 20mm balloon inflation showing partial angiographic compromise of the left main coronary artery.

FIGURE D: Angiographic image showing Palmaz stent inside of the CP stent to create the neoconduit. Angiography reveals widely patent left main coronary artery following neoconduit creation.

FIGURE E: Final pulmonary angiography following transcatheter valve implantation revealing no significant pulmonic regurgitation.

FIGURE F: Right ventricular pressure tracing obtained at the conclusion of the transcatheter pulmonic valve implantation procedure.

FIGURE G: Aortic root angiography performed pre- and post-TAVR. Image 1 arrows highlight significant source of perivalvular regurgitation. Image 2 showing final angiography with resolution of perivalvular leak 
after Sapien 3 Ultra implantation followed by bioprosthetic valve fracturing using a $22 \mathrm{~mm}$ Tru Dilatation balloon (BARD).

Figure H: Hemodynamic tracings obtained pre- and post-TAVR. The peak-to-peak gradient across the aortic valve went from $45 \mathrm{mmHg}$ to $1 \mathrm{mmHg}$, and the systemic diastolic pressure increased from $20 \mathrm{mmHg}$ to 60 $\mathrm{mmHg}$ following transcatheter aortic valve-in-valve implantation followed by bioprosthetic valve fracturing.

FIGURE I: Transesophageal echocardiographic images using color doppler performed pre- and post-TAVR showing near total resolution of significant perivalvular leak.

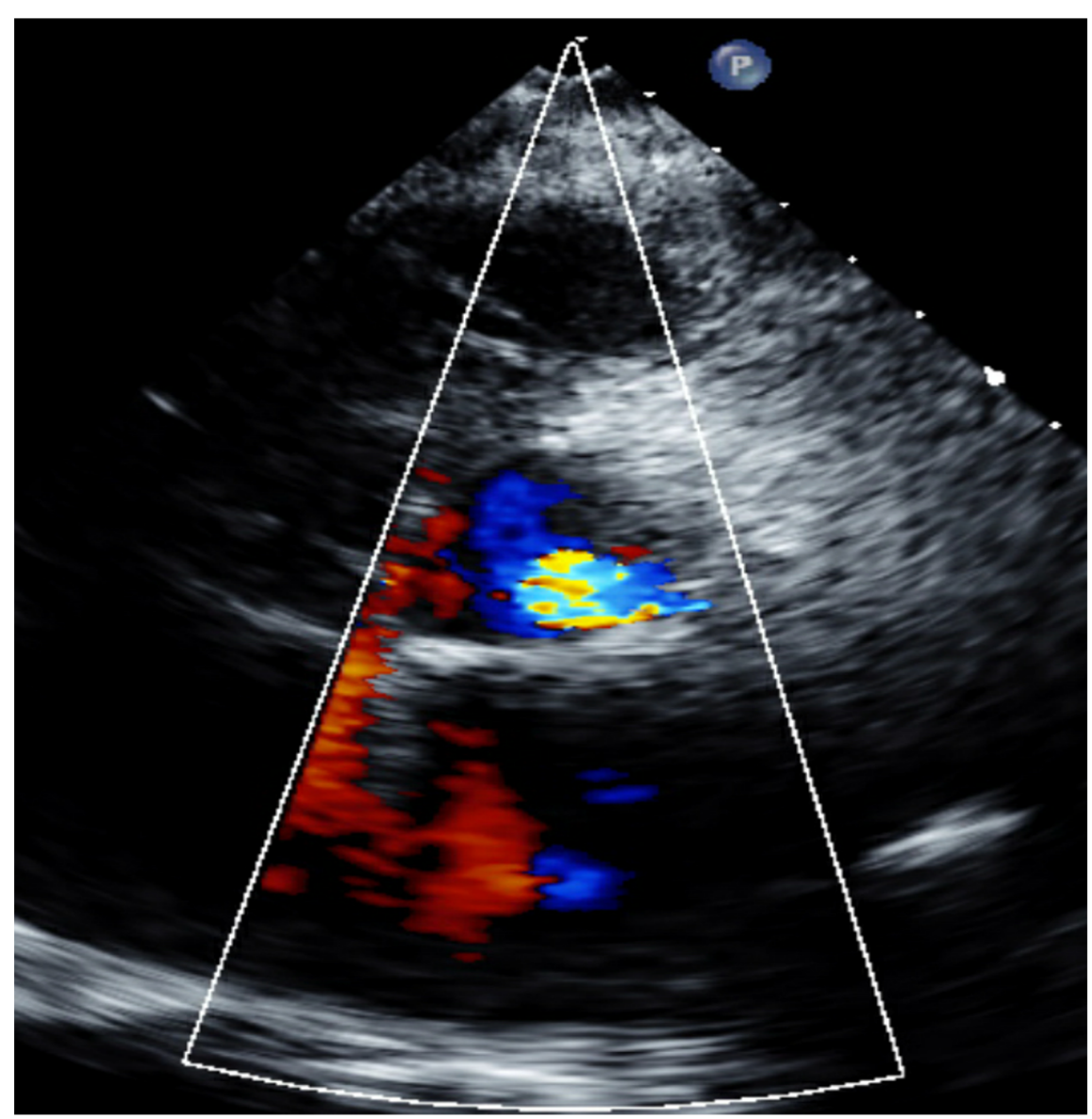



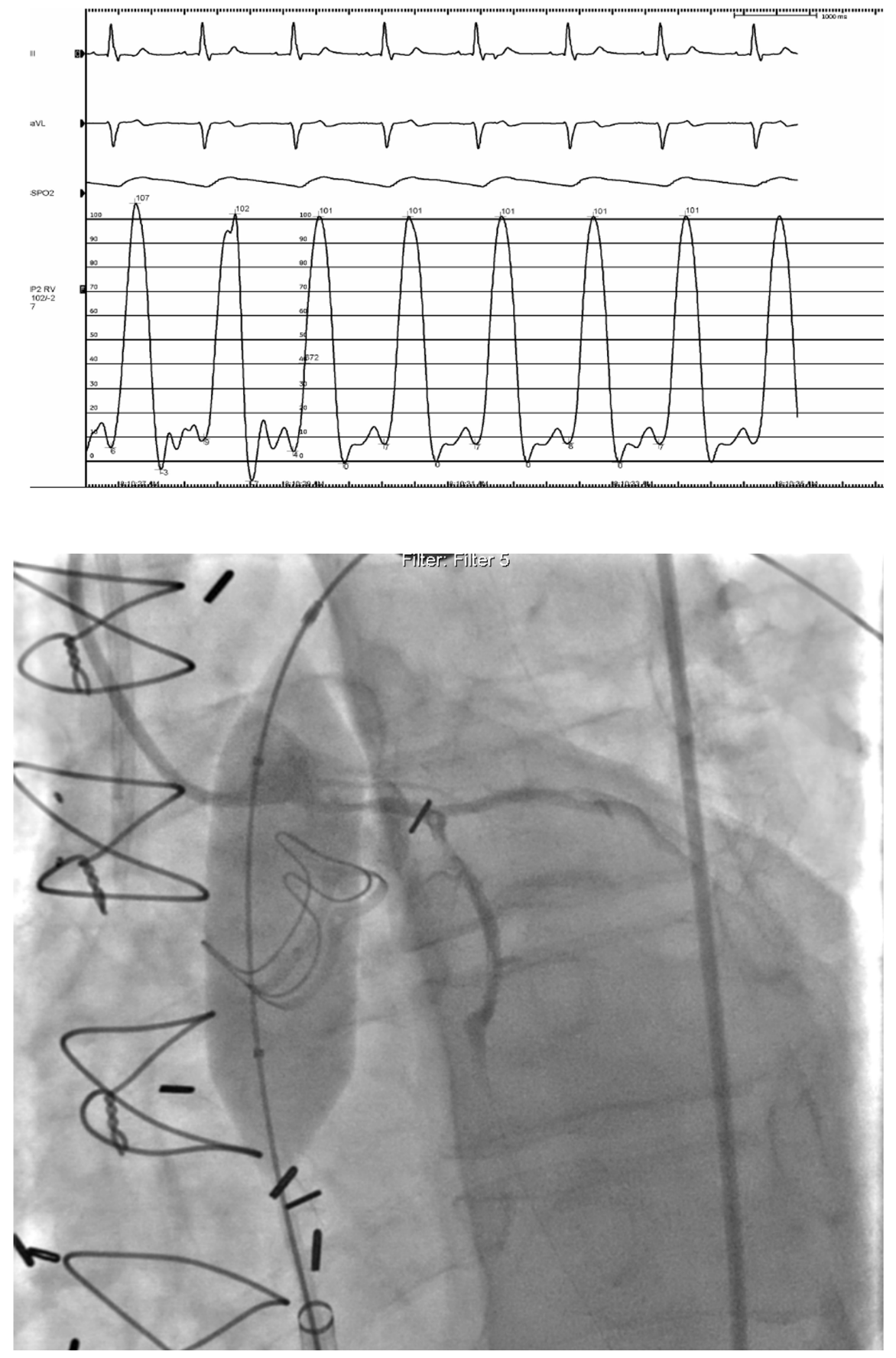


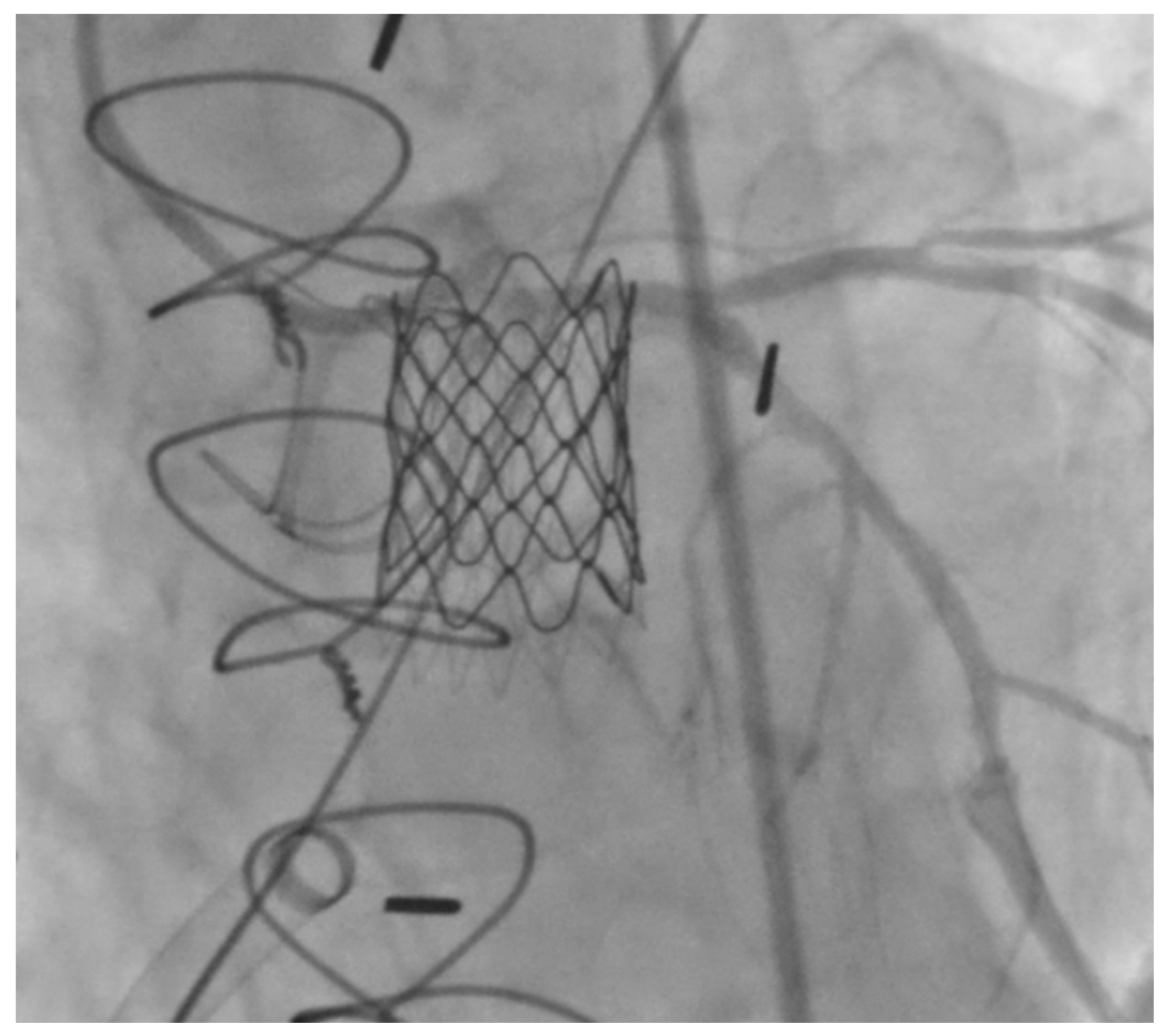



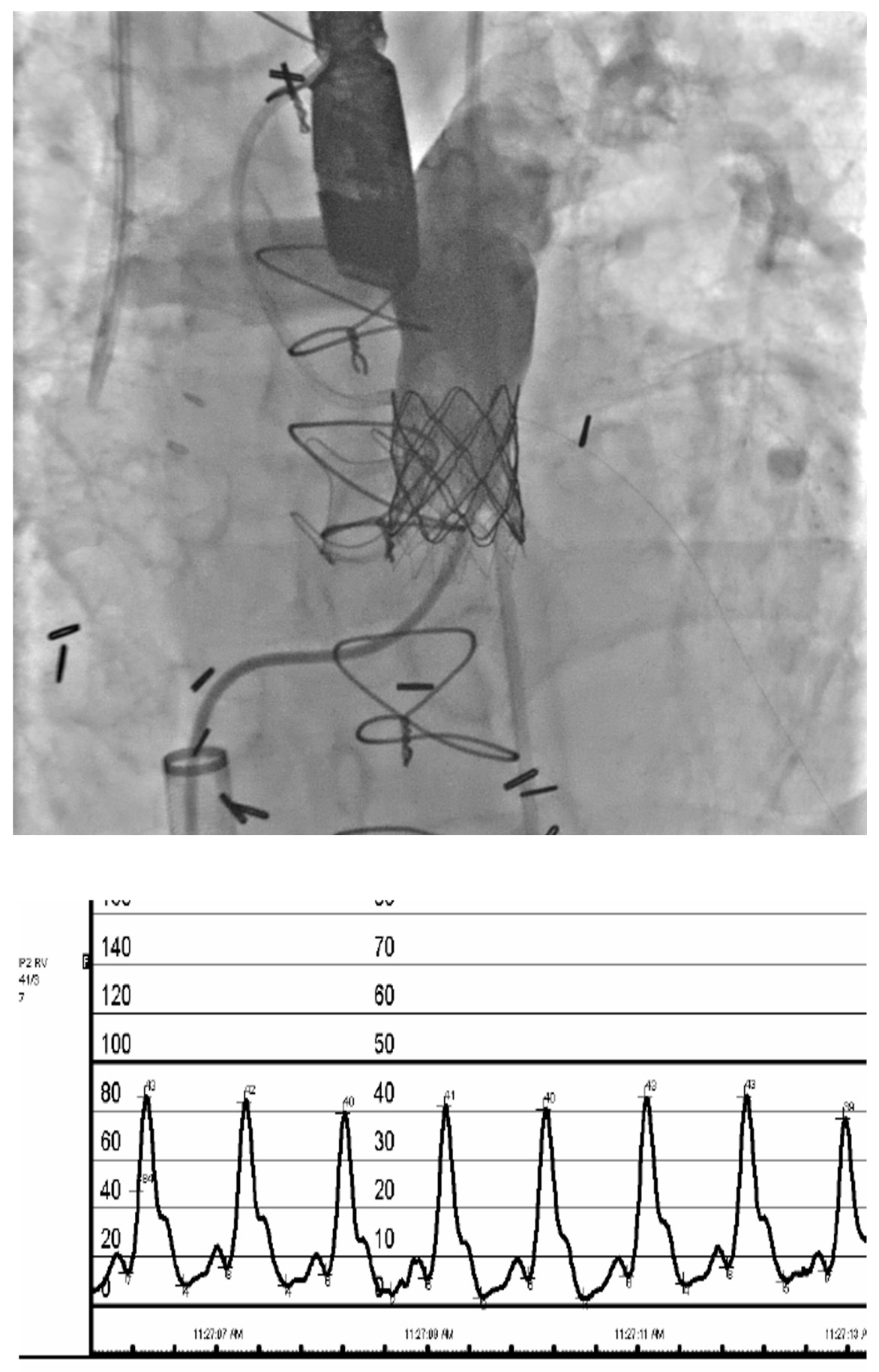


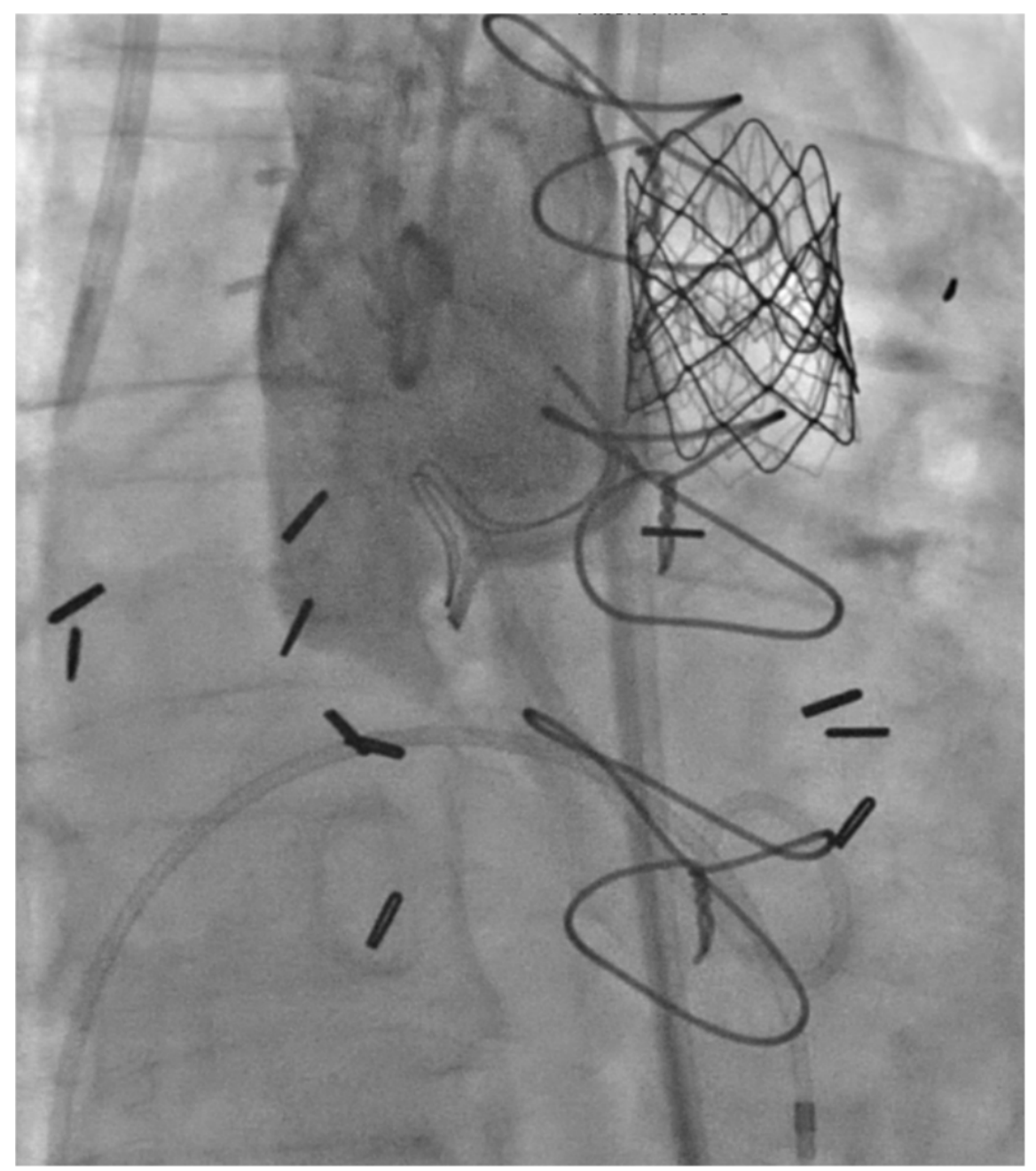



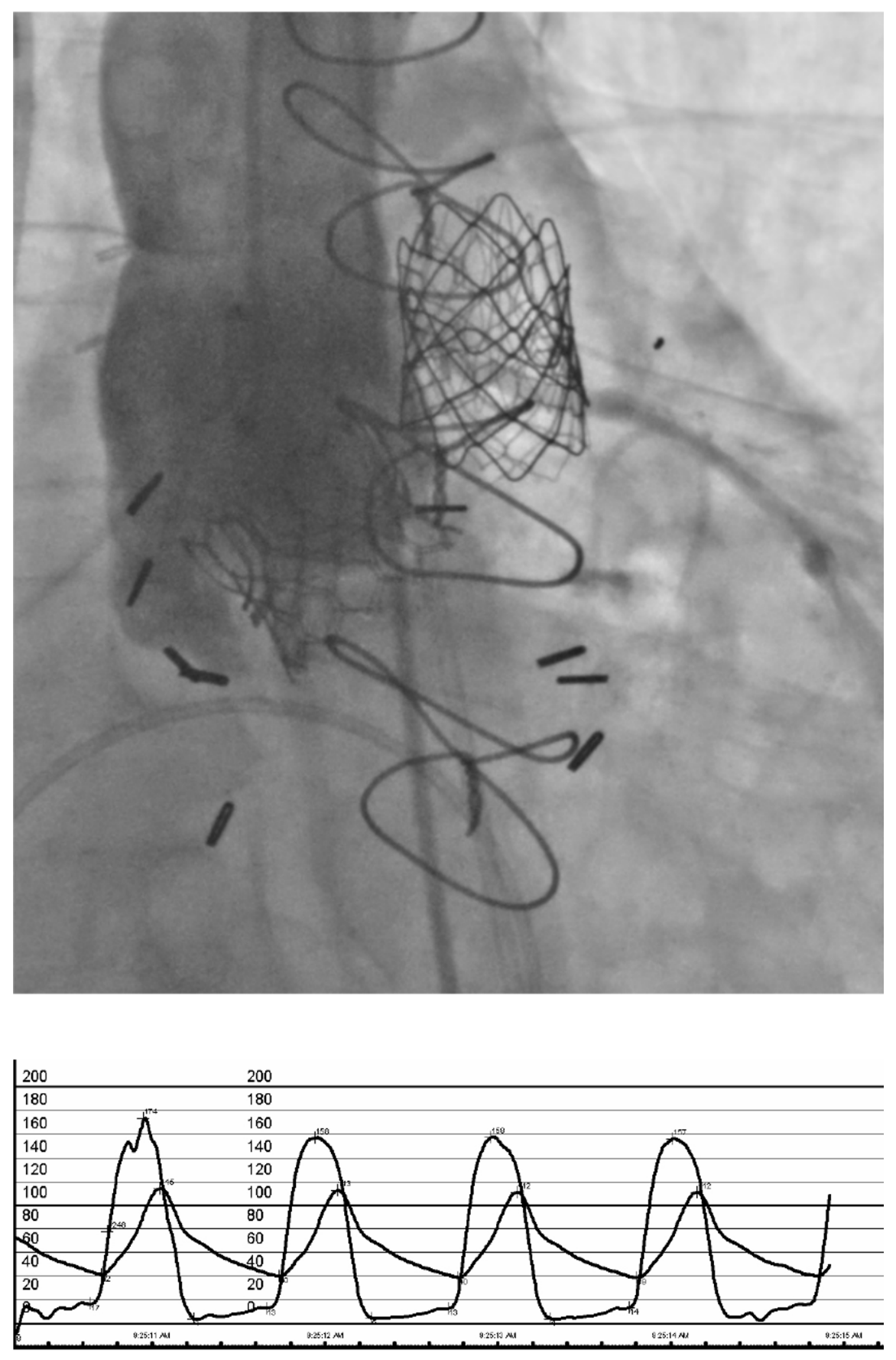

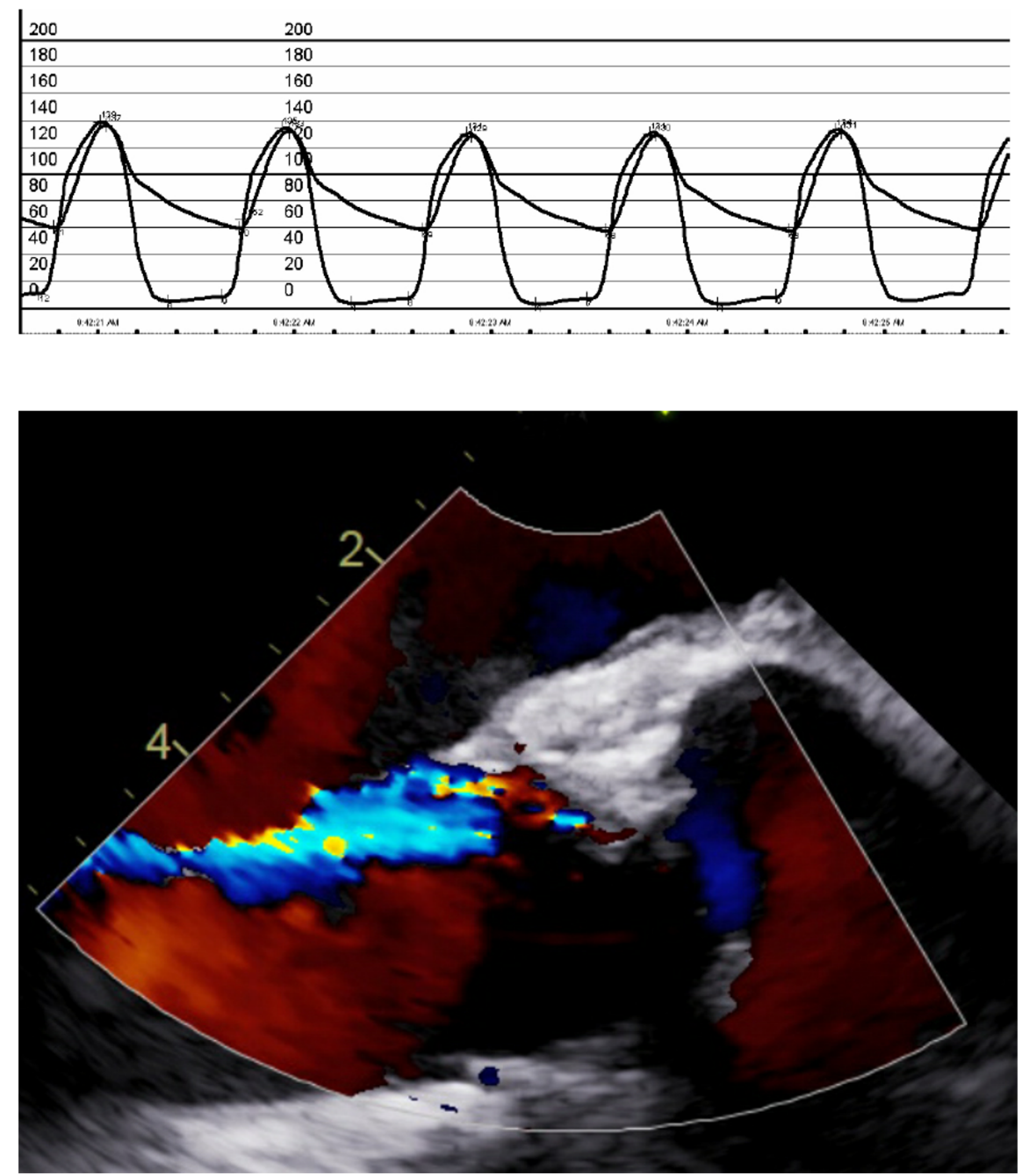


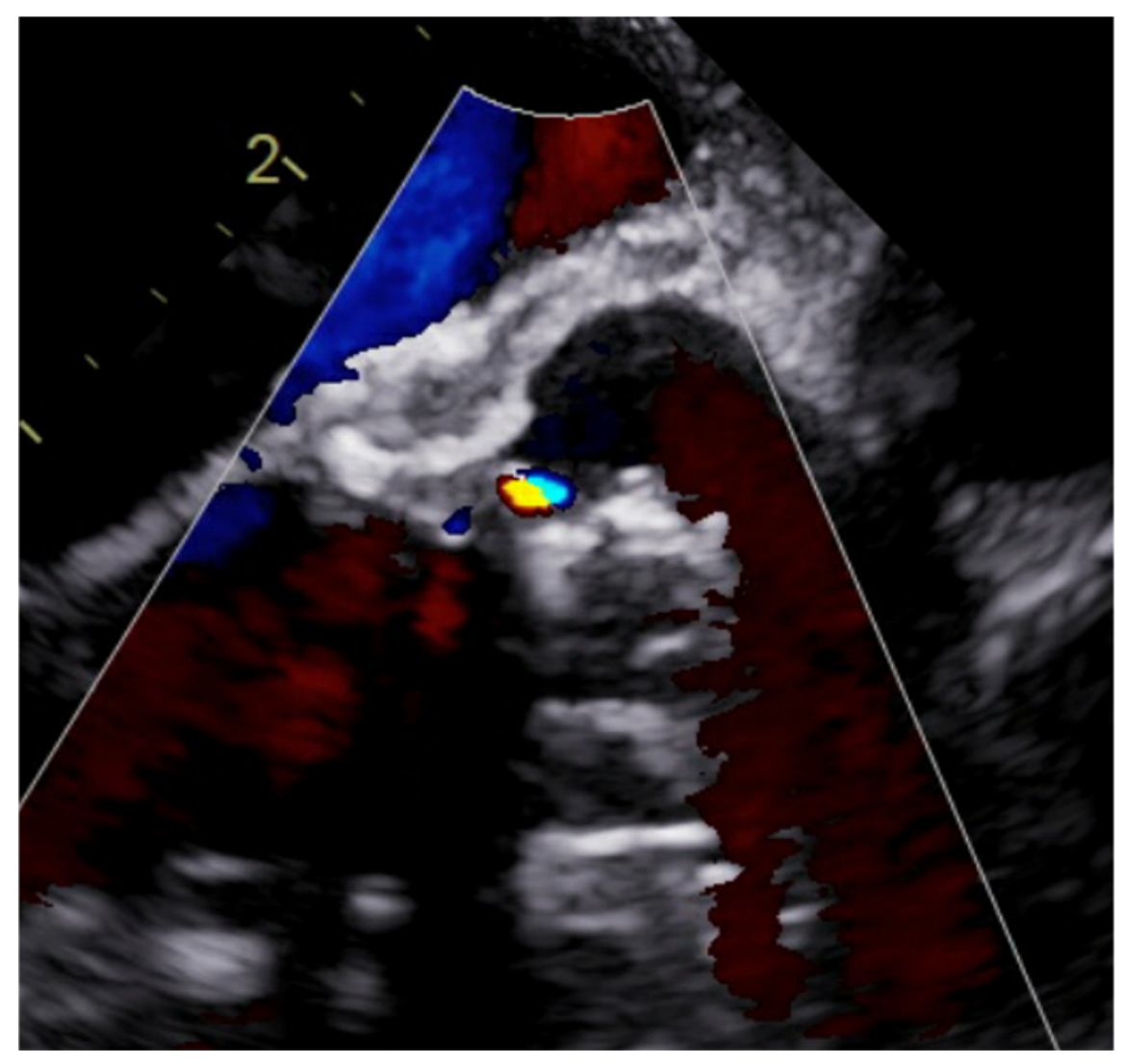

